\title{
APP: what's on the inside matters
}

Cleavage of the amyloid precursor protein (APP) at the cell surface produces extracellular amyloid- $\beta$ peptides $(\mathrm{A} \beta)$ and the APP intracellular domain, AICD. The predominant hypotheses in the Alzheimer's disease (AD) field have traditionally focused

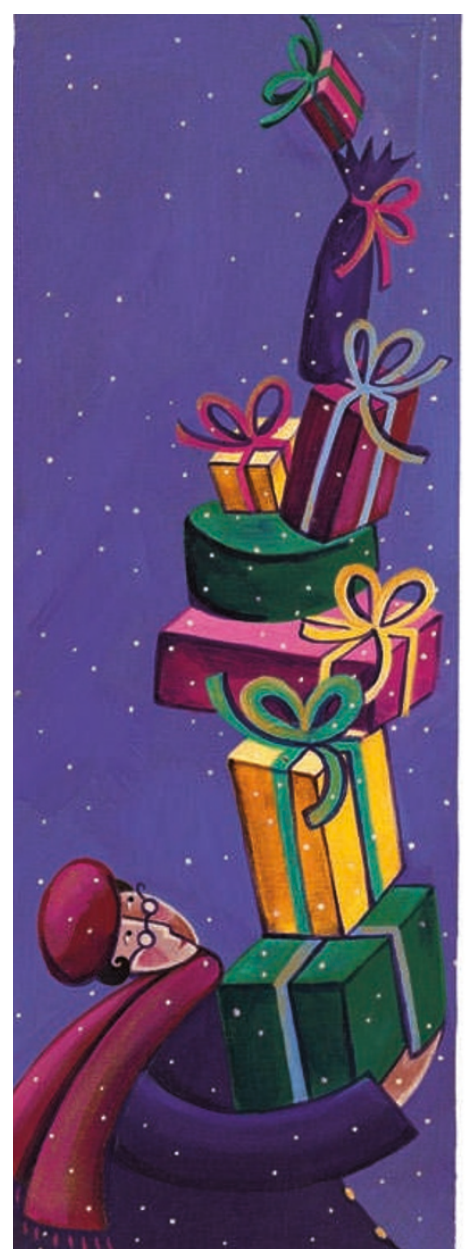

on a causative role for $\mathrm{A} \beta$; however, a new study by Pimplikar and colleagues points to a contribution of AICD to disease pathogenesis.

Several observations cannot be explained by the 'amyloid hypothesis', such as the poor correlation between $\mathrm{A} \beta$ deposition and disease severity, calling for the inclusion of additional contributing factors in any $\mathrm{AD}$ hypothesis. Here, the authors examined in detail the phenotype of a line of transgenic mice that overexpress AICD, revealing a number of remarkable similarities to human $\mathrm{AD}$.

The presence of aggregates of hyperphosphorylated tau protein is a classic hallmark of $\mathrm{AD}$. The authors found that phosphorylated tau levels were elevated in the AICDoverexpressing mice by 4 months of age and that tau aggregation was present by 8 months, mimicking the age-dependent changes seen in human AD. There was no evidence of altered APP processing or increased $A \beta$ levels or plaques resulting from AICD overexpression, suggesting that these effects on tau are not secondary to changes in $A \beta$ production.

Even more than tau aggregation, age-dependent neuron loss is the defining feature of AD. The authors observed greater loss of hippocampal neurons after 18 months of age in AICD-overexpressing mice than in controls. The brains of the transgenic mice were also more vulnerable to toxic insults: kainate-induced excitotoxicity at 4 months of age caused more hippocampal neuron loss in the mice overexpressing AICD than it did in controls.

In a Y-maze task, mice with normal working memory will typically remember the arm previously visited and thus tend to alternate between the two arms available for exploration in successive tasks. The AICD-overexpressing mice exhibited reduced alternation at 8 months of age, suggesting that — like patients with $\mathrm{AD}$ - their memory function is impaired.

These results showed that overexpression of AICD mimics many of the pathological changes observed in $\mathrm{AD}$, but are AICD levels altered in $\mathrm{AD}$ ? The authors found evidence for such elevated AICD concentrations in the brains of patients with $\mathrm{AD}$ compared with age-matched controls, implying that the findings may have clinical significance.

These findings suggest that increased AICD levels might have a role in $\mathrm{AD}$ pathology, although it is unknown whether this role is more or less significant than that of $A \beta$ accumulation or whether the two pathways work synergistically to cause disease. Nevertheless, one implication of the findings is that those developing strategies to combat $\mathrm{AD}$ should consider targeting AICD in addition to $A \beta$.

Katherine Whalley

ORIGINAL RESEARCH PAPER Ghosal, K. et al. Alzheimer's disease-like pathological features in transgenic mice expressing the APP intracellular domain. Proc. Natl Acad. Sci. USA 106, 18367-18377 (2009) 